\title{
Application of Nontraditional Optimization Techniques for Airfoil Shape Optimization
}

\author{
R. Mukesh, ${ }^{1}$ K. Lingadurai, ${ }^{1}$ and U. Selvakumar ${ }^{2}$ \\ ${ }^{1}$ Department of Mechanical Engineering, Anna University, Tamil Nadu, Dindigul 624622, India \\ ${ }^{2}$ Department of Information Technology, IBBT, Ghent University, 9050 Ghent, Belgium
}

Correspondence should be addressed to R. Mukesh, pr.mukeshphd@gmail.com

Received 16 May 2012; Accepted 21 September 2012

Academic Editor: Antonio Munjiza

Copyright (C) 2012 R. Mukesh et al. This is an open access article distributed under the Creative Commons Attribution License, which permits unrestricted use, distribution, and reproduction in any medium, provided the original work is properly cited.

\begin{abstract}
The method of optimization algorithms is one of the most important parameters which will strongly influence the fidelity of the solution during an aerodynamic shape optimization problem. Nowadays, various optimization methods, such as genetic algorithm (GA), simulated annealing (SA), and particle swarm optimization (PSO), are more widely employed to solve the aerodynamic shape optimization problems. In addition to the optimization method, the geometry parameterization becomes an important factor to be considered during the aerodynamic shape optimization process. The objective of this work is to introduce the knowledge of describing general airfoil geometry using twelve parameters by representing its shape as a polynomial function and coupling this approach with flow solution and optimization algorithms. An aerodynamic shape optimization problem is formulated for NACA 0012 airfoil and solved using the methods of simulated annealing and genetic algorithm for 5.0 deg angle of attack. The results show that the simulated annealing optimization scheme is more effective in finding the optimum solution among the various possible solutions. It is also found that the SA shows more exploitation characteristics as compared to the GA which is considered to be more effective explorer.
\end{abstract}

\section{Introduction}

The computational resources and time required to solve a given problem have always been a problem for engineers for a long time though a sufficient amount of growth is achieved in the computational power in the last thirty years. This becomes more complicated to deal with when the given problem is an optimization problem which requires huge amount of computational simulations. These kinds of problems have been one of the important problems to be addressed in the context of design optimization for quite some years. When the number of result(s) influencing variables are large in a given optimization problem, the required computational time per simulation increases automatically. This will severely influence the required computational resources to solve the given design optimization problem. Due to this reason, a need arises to describe a general geometry with minimum number of design variables. This leads to a search activity of finding some of the best parameterization methods. Nowadays, various parameterization methods are employed: partial differential equation approach (time consuming and not suitable for multidisciplinary design optimization), discrete points approach (the number of design variables becomes large), and polynomial approach (the number of design parameters depends on the degree of the polynomial chosen and suitable for multidisciplinary design optimization) are the three basic approaches to describe the geometry of a general airfoil [1-3]. Previous research works in design optimization suggest that the parameterization schemes highly influence the final optimum design which is obtained as a result of the optimization [4]. In this work, the parametric section (PARSEC) parameterization scheme is employed. The panel method is used to compute the flow field around the airfoil geometry during the design optimization process. Both SA and GA are employed to carry out the design optimization problem. This is not the first time that the mentioned optimization schemes (GA and SA) have been applied for the airfoil shape optimization. Here, in the current work, the capability of the strategies is investigated while they are applied to the airfoil kind of surfaces. Three 


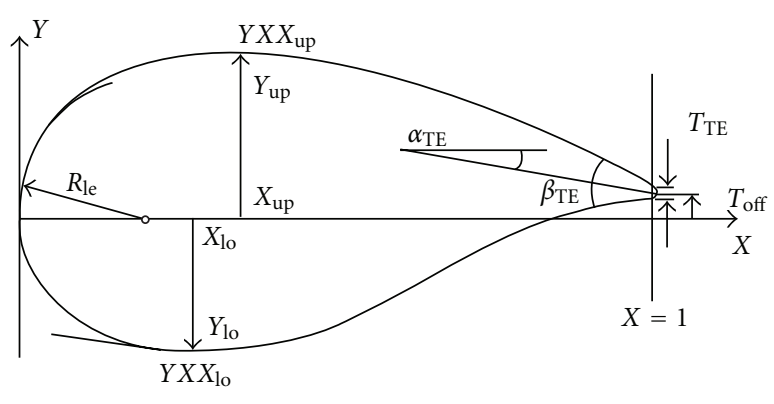

FIGURE 1: Control variables for PARSEC.

MATLAB codes are developed to implement PARSEC, panel, and SA approaches. A freely available FORTRAN code is picked for the GA. The results and issues faced during the whole design process are discussed in the following sections.

\section{PARSEC}

In PARSEC parametrisation scheme, an unknown linear combination of suitable base functions is used to describe the airfoil geometry [5]. This approach is considered to be more suitable for design optimization problems, since the geometric constraints on the airfoil shape can be described by some simple linear constraints. Twelve design variables are chosen to have direct control over the shape of the airfoil. The twelve design variables are upper leading edge radius $\left(R_{\text {leu }}\right)$, lower leading edge radius $\left(R_{\text {lel }}\right)$, upper crest point $\left(Y_{\text {up }}\right)$, lower crest point $\left(Y_{\mathrm{lo}}\right)$, position of upper crest $\left(X_{\mathrm{up}}\right)$, position of lower crest $\left(X_{\mathrm{lo}}\right)$, upper crest curvature $\left(Y X X_{\text {up }}\right)$, lower crest curvature $\left(Y X X_{\mathrm{lo}_{\mathrm{o}}}\right)$, and trailing edge offset $\left(T_{\mathrm{off}}\right)$, trailing edge thickness $\left(T_{\mathrm{TE}}\right)$, trailing edge direction angle $\left(\alpha_{\mathrm{TE}}\right)$, and trailing edge wedge angle $\left(\beta_{\mathrm{TE}}\right)$, as shown in Figure 1. The leading edge radius parameters provide more control at the leading edge of the airfoil geometry. The mathematical relations for the PARSEC approach are given as follows:

$$
\begin{aligned}
& y_{u}=\sum_{i=1}^{6} a_{i} x^{i-(1 / 2),} \\
& y_{l}=\sum_{i=1}^{6} b_{i} x^{i-(1 / 2)},
\end{aligned}
$$

where $y_{u}$ is the upper $y$ coordinate, $y_{l}$ is the lower $y$ coordinate, and $a_{i}, b_{i}$ are the unknown coefficients to be solved from the specified values of the twelve design variables. The previous polynomial equations are solved using a set of geometrical conditions.

\section{Panel Technique}

The panel method is used to solve the potential equations without being computationally expensive. It provides more reasonably accurate results. These two properties make the panel method to be more suitable for design optimization problems where the number of simulations is incredibly large. Since the current problem deals with the incompressible subsonic flow region, this approach is employed in this

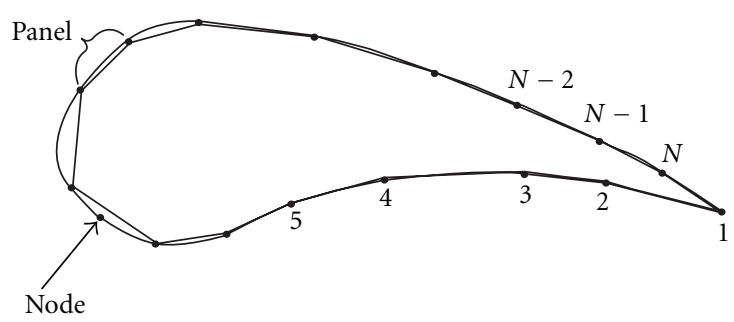

Figure 2: Nodes and Panels.

work. The solution procedure for panel technique consists of discretizing the surface of the airfoil into straight line segments or panels and assuming the following conditions: (a) the source strength is constant over each panel but has a different value for each panel and (b) the vortex strength is constant and equal over each panel $[6,7]$. The compressibility and the viscosity of air in the flow field are neglected. But it is required to satisfy the condition that the net viscosity of the flow should be such that the flow leaving the trailing edge is smooth. The curl of the velocity field is assumed to be zero. Hence,

$$
\phi=\phi_{\infty}+\phi_{\delta}+\phi_{v}
$$

where $\phi$, which is expressed as a summation of the free stream potential, source potential, and vortex potential, is the total potential function. Except the free stream potential, the other potentials have potentially locally varying strengths. Figure 2 depicts the notations of the panel approach.

As the number of panels increases, the accuracy of the solution increases. Indeed, the computational time will increase as the number of panels increases. $N+1$ node points define $N$ panels. The tangential velocity $\left(V_{\mathrm{ti}}\right)$ at the centre of each panel is estimated by imposing a flow tangency condition at each panel. The coefficient of pressure $\left(C_{p}\right)$ at each panel is calculated using the following relation:

$$
C_{p}\left(x_{i}, y_{i}\right)=1-\left[\frac{V_{\mathrm{ti}}^{2}}{V_{\infty}^{2}}\right] \text {. }
$$

\section{Simulated Annealing}

Simulated Annealing [8, 9] is one kind of non-traditional based optimization algorithm for searching global optimum. It is a point-by-point method. It resembles the cooling process of molten metals through annealing, and the formation of the crystal depends upon the cooling rate. The process of slow cooling is called as annealing. The cooling phenomenon is simulated by controlling a temperature-like parameter, and it can be done by introducing the concept of Boltzmann probability distribution. In addition to that, Metropolis suggested one idea to implement the Boltzmann probability function in simulated systems for better optimization.

The main steps of simulated annealing are given as follows.

(a) Choose an initial point and a high temperature $T$.

(b) A second point is created at random in the vicinity of the initial point. 
(c) The difference between these two points is calculated.

(d) If the second point has a larger function value, the point is accepted.

(e) In the next generation, another point is created at random in the neighbourhood of the current point and the Metropolis algorithm $[10,11]$ is used to accept or reject the point.

(f) The algorithm is terminated when an optimized value is obtained.

The initial value (lower bound values) and the number of iterations $\left(1 \times 10^{5}\right)$ are the two important parameters of the simulated annealing. So, we have to choose these two parameters according to our optimization problem to be solved. The temperature is a controlling parameter which is used to find out the functional value (coefficient of lift) from the given points (lower and upper bound values), and this process will be continued until the optimized value (maximum vertical aerodynamic force) is obtained.

\section{Genetic Algorithm}

Genetic algorithms (GA), in contrast to gradient optimization approaches, offer an alternative approach with several attractive features. The basic idea associated with the GA is to search for optimal solutions using an analogy to the theory of evolution. During solution advance (or "evolution" using GA terminology), each chromosome is ranked according to its fitness vector-one fitness value for each objective. The higher ranking chromosomes are selected to continue to the next generation while the probability of the selection of lower ranking chromosomes is less. In every generation, a new set of artificial creatures (strings) is created using bits and pieces of the fittest of the old; an occasional new part is tried for good measure. While randomized, genetic algorithms are not simple random walk. They efficiently exploit historical information to speculate on new search points with expected improved performance. The newly selected chromosomes in the next generation are manipulated using various operators (combination, crossover, or mutation) to create the final set of chromosomes for the new generation. These chromosomes are then evaluated for fitness, and the process continues iterating from generation to generation-until a suitable level of convergence is obtained or until a specified number of generations have been completed. GA optimization requires no gradients; it does not need the sensitivity of derivatives. It theoretically works well in nonsmooth design spaces containing several or perhaps many local extrema. It is also an attractive method for multiobjective design optimization applications offering the ability to compute the so-called "pareto-optimal sets" instead of the limited single design point traditionally provided by other methods. The basic genetic algorithm comprises four important steps. They are initialisation, selection, crossover, and mutation $[12,13]$.

In GA the PARSEC parameterization scheme variables act as the optimization parameters (design variables) which will influence the coefficient of lift (objective function). A pool of optimization parameters will be generated by the
TABLe 1: Optimization objectives and constraints.

\begin{tabular}{ll}
\hline $\begin{array}{l}\text { Angle of attack } \\
\text { Flow constraint }\end{array}$ & $\begin{array}{l}5.0 \mathrm{deg} \\
\text { Subsonic and incompressible } \\
\text { Geometric constraint }\end{array}$ \\
$\begin{array}{l}\text { Max thickness must be less than } 10 \% \\
\text { chord length } \\
T_{\mathrm{TE}} \text { and } T_{\text {off }} \text { the airfoil is zero }\end{array}$ \\
$\begin{array}{l}\text { Aerodynamic constraint } \\
\text { Objective }\end{array}$ & $\begin{array}{l}\text { Lift not less than the original one } \\
\text { Maximize coefficient of lift }\end{array}$ \\
\hline
\end{tabular}

GA within the defined range of values of the optimization parameters to start the optimization process. Then, based on two GA operators, crossover and mutation, the best optimization parameters at each generation which will increase the coefficient of lift will be selected. This process will be continued until the whole design space is completely explored.

\section{Optimization of NACA 0012 Airfoil}

The aerodynamic shape optimization process is carried out with an intention of increasing the vertical aerodynamic force subject to aerodynamic and structural constraints. The structural constraints are implemented by fixing the values of trailing edge thickness and trailing edge offset parameters during the optimization in both of the optimization schemes. These constraints are placed in order to avoid the optimizer to get converged at inefficient locations and to avoid getting unrealistic aerodynamic shapes. Since the panel method is only applicable for low speed flows, a flow constraint is placed to keep the assumptions valid throughout the whole optimization process. The flow constraint is implemented by fixing the angle of attack at $5.0 \mathrm{deg}$. For each design parameter, lower and upper bound values are defined. Each generation produced by the SA and genetic algorithms has the best set of twelve PARSEC parameters. The corresponding airfoil profile is generated using PARSEC parametrisation. Then, the panel method is used to compute the flow around the airfoil at 5.0 deg angle of attack. From the pressure distribution, the lift coefficient is calculated using the trapezoidal rule. This new coefficient of lift is compared to the original one. The SA and genetic algorithms in the end will lead to the best set of PARSEC parameters which will maximise the objective function within the search space. The design conditions, optimization objectives and constraints, which are used during the optimization process using SA and GA, are tabulated in Table 1.

\section{Result and Analysis}

The initial PARSEC parameters have been given approximately by specifying their lower and upper bound values. There is no need for specifying this accurately. The geometry of the airfoil is expressed by the best twelve PARSEC parameters resulting from the SA algorithm which exhibits a considerable increase in the coefficient of lift as compared to the best solution found by the genetic algorithm. There is a 


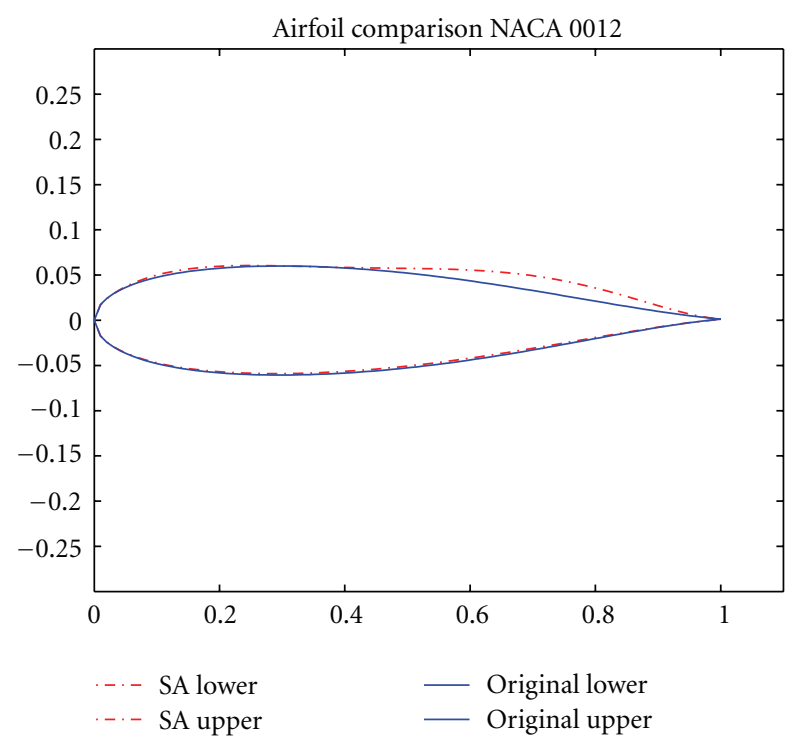

FIGURE 3: Original NACA 0012 airfoil versus optimized airfoil using SA.

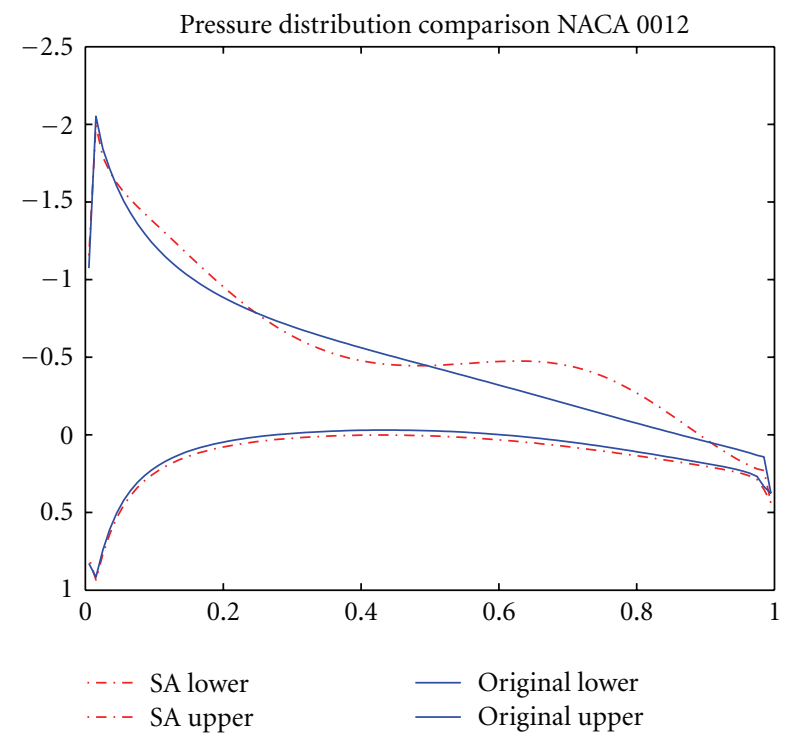

FIGURE 4: Comparison of pressure distribution over the surface of original NACA 0012 airfoil and optimized airfoil using SA.

history for the SA to be good for problems involving highly nonlinear functions where the function has large number of peaks and valleys. It is again witnessed from the obtained results that the SA has not got stuck with the local optima or extrema. The comparison between the original NACA 0012 airfoil geometry and the optimized airfoil geometry using SA is indicated in Figure 3. The comparison of pressure distribution over the surface of the original NACA 0012 airfoil and the optimized airfoil using SA is shown in Figure 4. It can be seen from these figures that the actual airfoil geometry is modified in such a way that the airflow is highly accelerated in the upper surface of the optimized airfoil as compared to the actual airfoil. From this, it can be

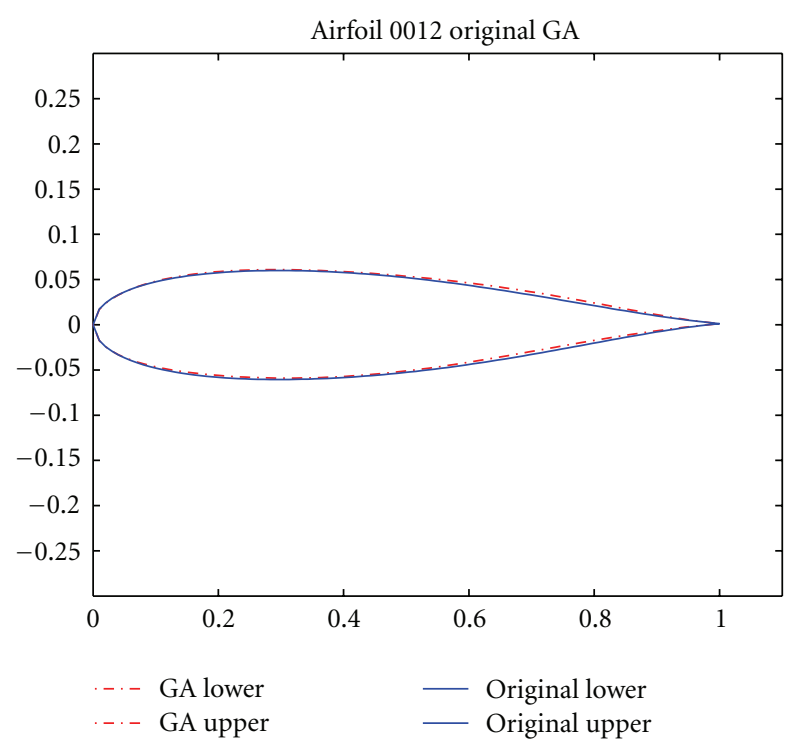

FIGURE 5: Comparison of original NACA 0012 airfoil and optimized airfoil using GA.

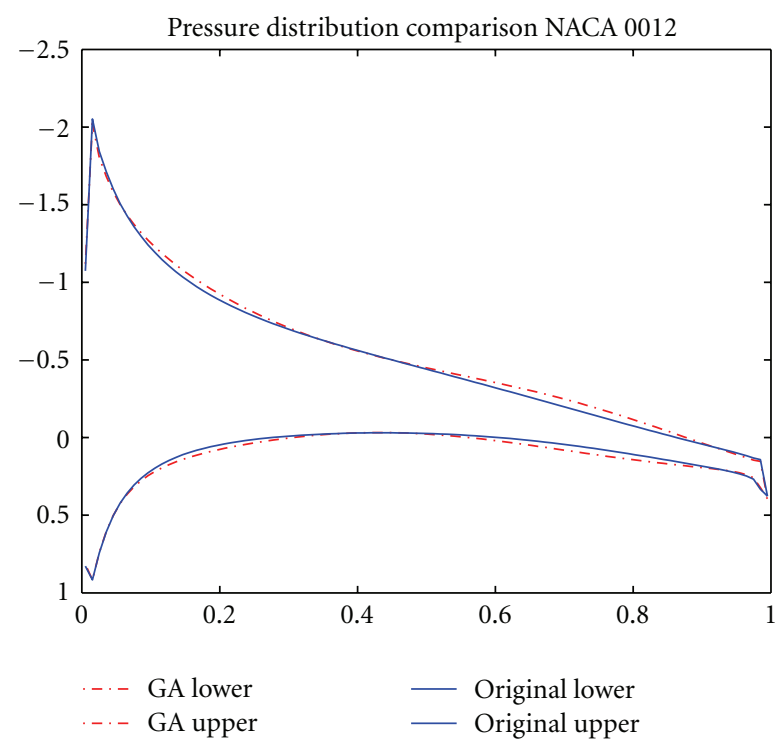

FIGURE 6: Comparison of pressure distribution over the surface of original NACA 0012 airfoil and optimized airfoil using GA.

clearly understood that the increase in the lift coefficient is caused by the pressure variation in the upper surface of the optimized airfoil. Figure 5 shows the comparison between the original NACA 0012 airfoil geometry and the optimized airfoil geometry found by GA. The comparison of pressure distribution over the surface of the original NACA 0012 airfoil and the optimized airfoil found by GA is given in Figure 6.

The comparison of geometry and its corresponding pressure distribution between the optimum designs which are found by both SA and GA is depicted in Figures 7 and 8 respectively. It can be clearly seen that the variation of the geometry found by the GA is quite less compared to the SA, 
TABLE 2: Optimized PARSEC parameters.

\begin{tabular}{lccc}
\hline Parameter & Original value & Optimized value using SA & Optimized value using GA \\
\hline$\left(R_{\mathrm{leu}}\right)$ upper leading edge radius & 0.0155 & 0.0140 & 0.0145 \\
$\left(R_{\mathrm{lel}}\right)$ lower leading edge radius & 0.0155 & 0.0152 & 0.0160 \\
$\left(X_{\mathrm{up}}\right)$ position of upper crest & 0.296632 & 0.2500 & 0.2900 \\
$\left(Y_{\mathrm{up}}\right)$ upper crest point & 0.060015 & 0.0605 & 0.0610 \\
$\left(Y X X_{\mathrm{up}}\right)$ upper crest curvature & -0.4515 & -0.4600 & -0.4480 \\
$\left(X_{\mathrm{lo}}\right)$ position of lower crest & 0.296632 & 0.2900 & 0.3100 \\
$\left(Y_{\mathrm{lo}}\right)$ lower crest point & -0.06055 & -0.0590 & -0.0590 \\
$\left(Y X X_{\mathrm{lo}}\right)$ lower crest curvature & 0.453 & 0.4588 & 0.4599 \\
$\left(T_{\mathrm{TE}}\right)$ trailing edge thickness & 0 & 0 & 0 \\
$\left(T_{\mathrm{off}}\right)$ trailing edge offset & 0.001260 & 0.0011 & 0.0012 \\
$\left(\alpha_{\mathrm{TE}}\right)$ trailing edge direction angle & 0 & 0 & 0 \\
$\left(\beta_{\mathrm{TE}}\right)$ trailing edge wedge angle & 7.36 & 7.300 & 7.2484 \\
\hline
\end{tabular}

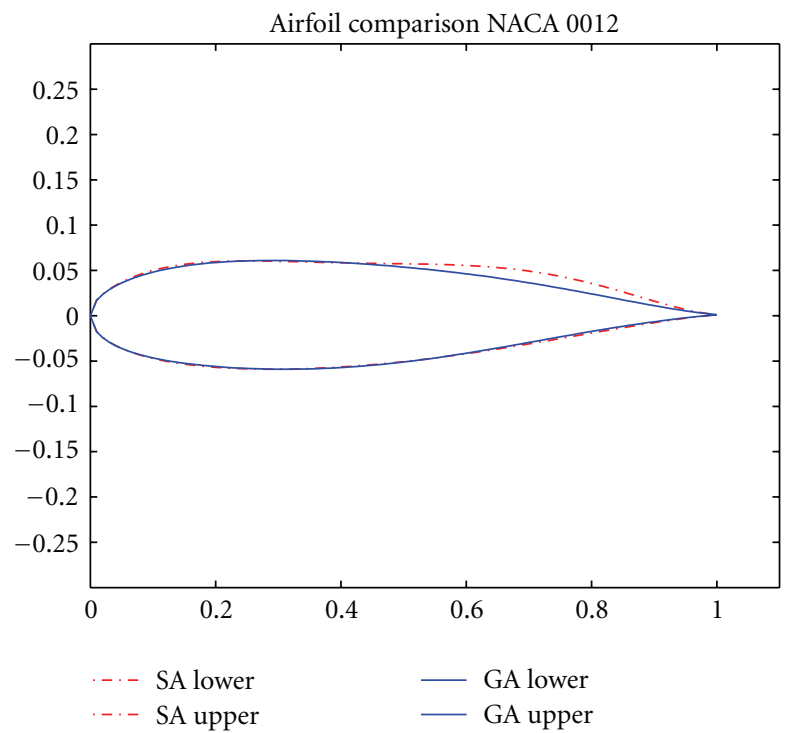

FIgURE 7: Comparison of optimized airfoil using both GA and SA.

though the same design space is given to them to be explored. It can also be noticed that the geometry found by SA has more negative pressure at the upper surface which is one of most important requirements for an efficient aerodynamic design. The optimized values of PARSEC parameters which are found by both GA and SA and their corresponding coefficient of lift values are tabulated and compared with the actual values in Tables 2 and 3, respectively. It can be clearly seen that airfoil geometry which is found by SA has more coefficient of lift as compared to the airfoil geometry which is found by GA.

\section{Conclusion}

A problem of optimizing the actual NACA 0012 airfoil geometry for the previously discussed flow and geometrical conditions are formulated and solved using two optimization schemes, simulated annealing and genetic algorithm. The optimized airfoil geometries have an improved coefficient of

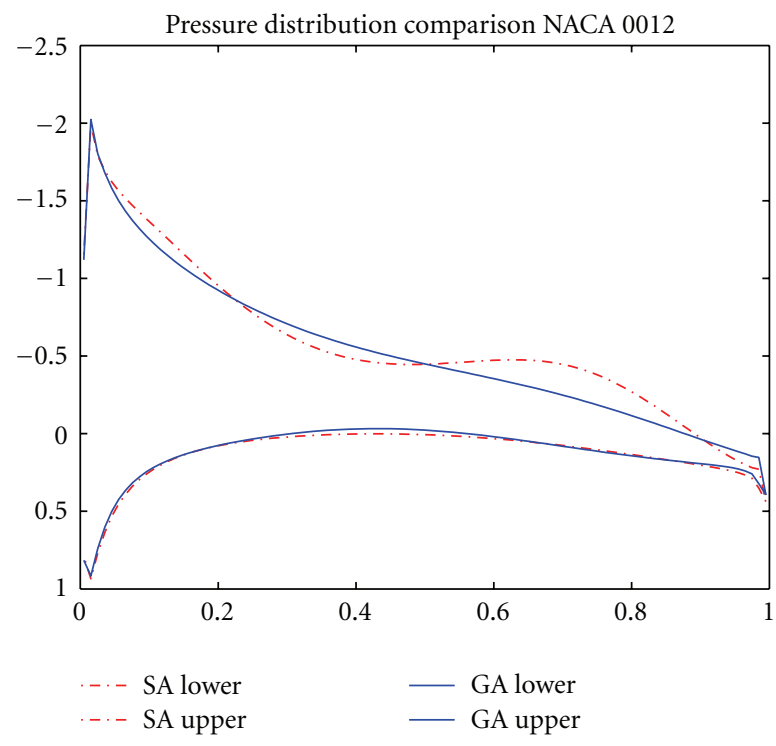

FIGURE 8: Comparison of pressure distribution over the surface of optimized airfoil using both GA and SA.

TABLE 3: Original versus optimized coefficient of lift.

\begin{tabular}{lccc}
\hline Angle of attack & $\mathrm{Cl}_{\text {original }}$ & $\mathrm{Cl}_{\text {optimized using SA }}$ & $\mathrm{Cl}_{\text {optimized using GA }}$ \\
\hline 5.0 & 0.55 & 0.69429 & 0.62571 \\
\hline
\end{tabular}

lift of 0.6942 (SA) and 0.6257 (GA) as compared to the actual NACA0012 airfoil geometry which has 0.55 at 5.0 deg angle of attack. The PARSEC parametrisation scheme is used to express the shape of the airfoil. The result shows that the PARSEC parameters show proper control over the aerodynamic performance of the airfoil by effectively controlling the aerodynamic shape of the airfoil. The PARSEC approach eases the way of understanding the impact of individual geometrical parameters on the aerodynamic properties of the airfoil. It is once again witnessed that the panel method gives reasonably accurate results without being computationally expensive. It is concluded from the results that the SA 
algorithm is so effective in finding the best solution among many possible solutions within a search space as compared to the GA optimization scheme in the current formulated problem. During the optimization process, plenty of airfoil data is obtained. It can be effectively used for the airfoil design by making use of these data for constructing mathematical models. The constructed mathematical models can be suitably applied to new design studies of innovative configurations.

\section{References}

[1] P. Castonguay and S. K. Nadarajah, "Effect of shape parameterization on aerodynamic shape optimization," in Proceedings of the 45th Aerospace Sciences Meeting and Exhibit (AIAA '07), pp. 561-580, January 2007.

[2] R. Balu and V. Ashok, Airfoil shape optimization using paras3D software and genetic algorithm VSSC/ARD/TR/095/2006, Vikram Sarabhai Space Centre, Kerala, India, 2006.

[3] G. S. Avinash and S. A. Lal, Inverse Design of Airfoil Using Vortex Element Method, Department of Mechanical Engineering, College of Engineering, Thiruvananthapuram, Kerala, India, 2010.

[4] R. Balu and U. Selvakumar, "Optimum hierarchical Bezier parameterization of arbitrary curves and surfaces," in Proceedings of the 11th Annual CFD Symposium, pp. 46-48, Indian Institute of Science, Bangalore, India, August 2009.

[5] H. Sobieczky, Parametric Airfoils and Wings, vol. 68 of Notes on Numerical Fluid Mechanics, Vieweg, 1998.

[6] J. L. Hess, "Panel methods in computational fluid dynamics," Annual Review of Fluid Mechanics, vol. 22, no. 1, pp. 255-274, 1990.

[7] J. Katz and A. Plotkin, Low-Speed Aerodynamics from Wing Theory to Panel Methods, McGraw-Hill, New York, NY, USA, 1991.

[8] B. Behzadi, C. Ghotbi, and A. Galindo, "Application of the simplex simulated annealing technique to nonlinear parameter optimization for the SAFT-VR equation of state," Chemical Engineering Science, vol. 60, no. 23, pp. 6607-6621, 2005.

[9] Margarida, F. Cardoso, R. L. Salcedo, and S. F. De Azevedo, "The simplex-simulated annealing approach to continuous non-linear optimization," Computers and Chemical Engineering, vol. 20, no. 9, pp. 1065-1080, 1996.

[10] N. Metropolis, A. W. Rosenbluth, M. N. Rosenbluth, A. H. Teller, and E. Teller, "Equation of state calculations by fast computing machines," The Journal of Chemical Physics, vol. 21, no. 6, pp. 1087-1092, 1953.

[11] S. Kirkpatrick, C. D. Gelatt, and M. P. Vecchi, "Optimization by simulated annealing," Science, vol. 220, no. 4598, pp. 671680, 1983.

[12] D. E. Goldberg, Genetic Algorithms in Search, Optimization and Machine Learning, Addison-Wesley, Reading, Mass, USA, 1989.

[13] R. Mukesh and U. Selvakumar, "Aerodynamic Shape Optimization using Computer Mapping of Natural Evolution Process," in Proceedings of the International Conference on Mechanical and Aerospace Engineering at University of Electronics Science and Technology of China, vol. 5, pp. 367-371, April 2010. 

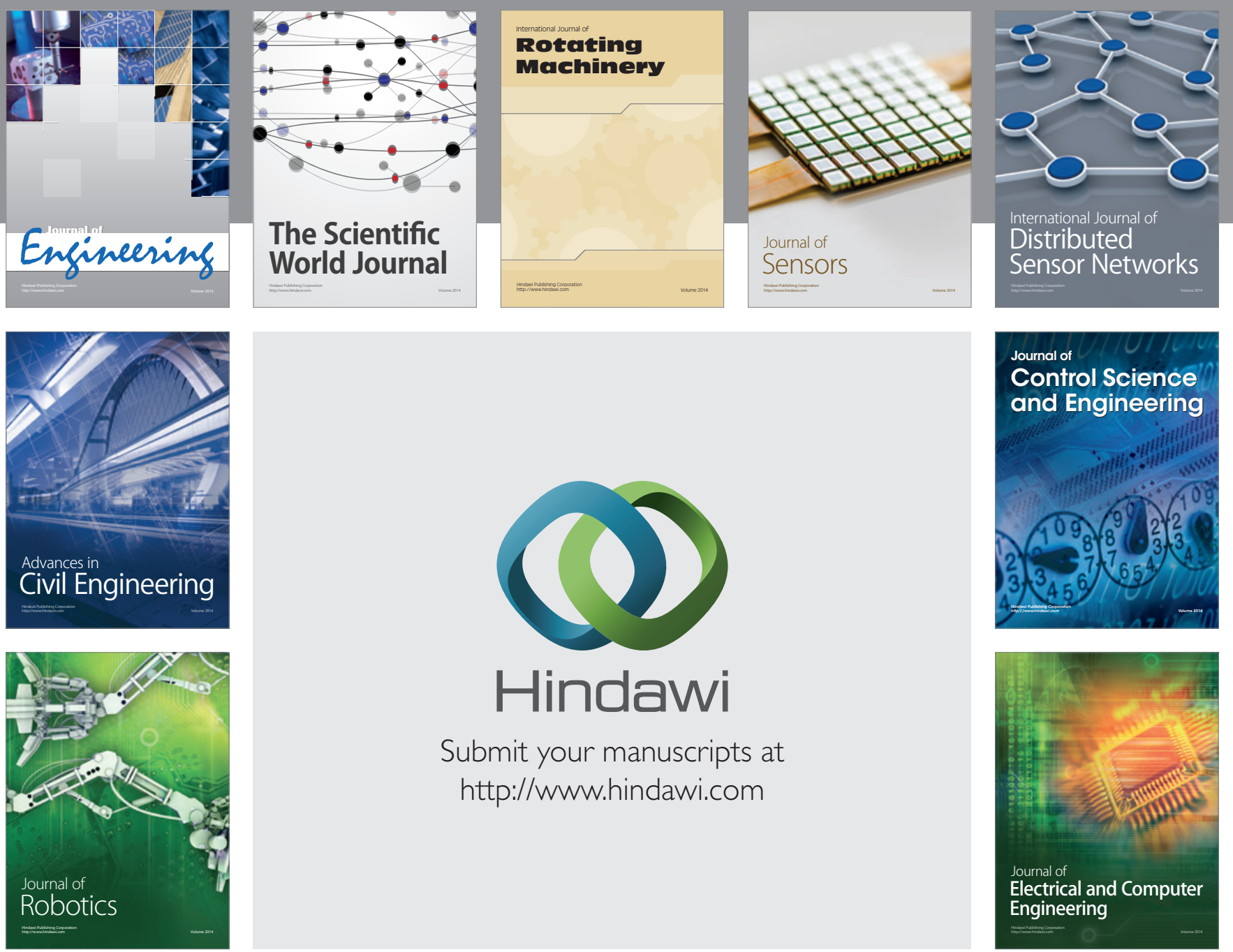

Submit your manuscripts at

http://www.hindawi.com
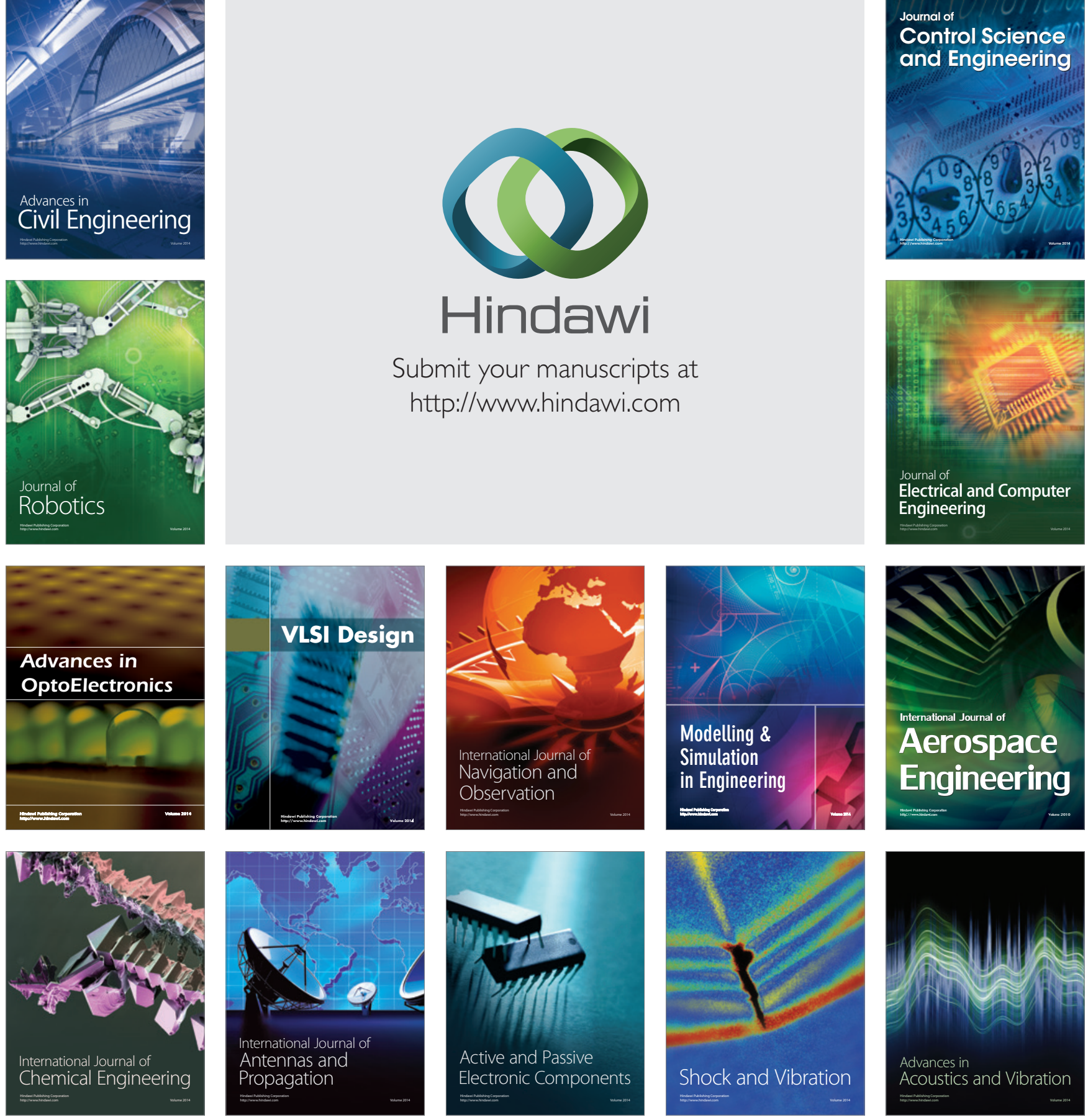